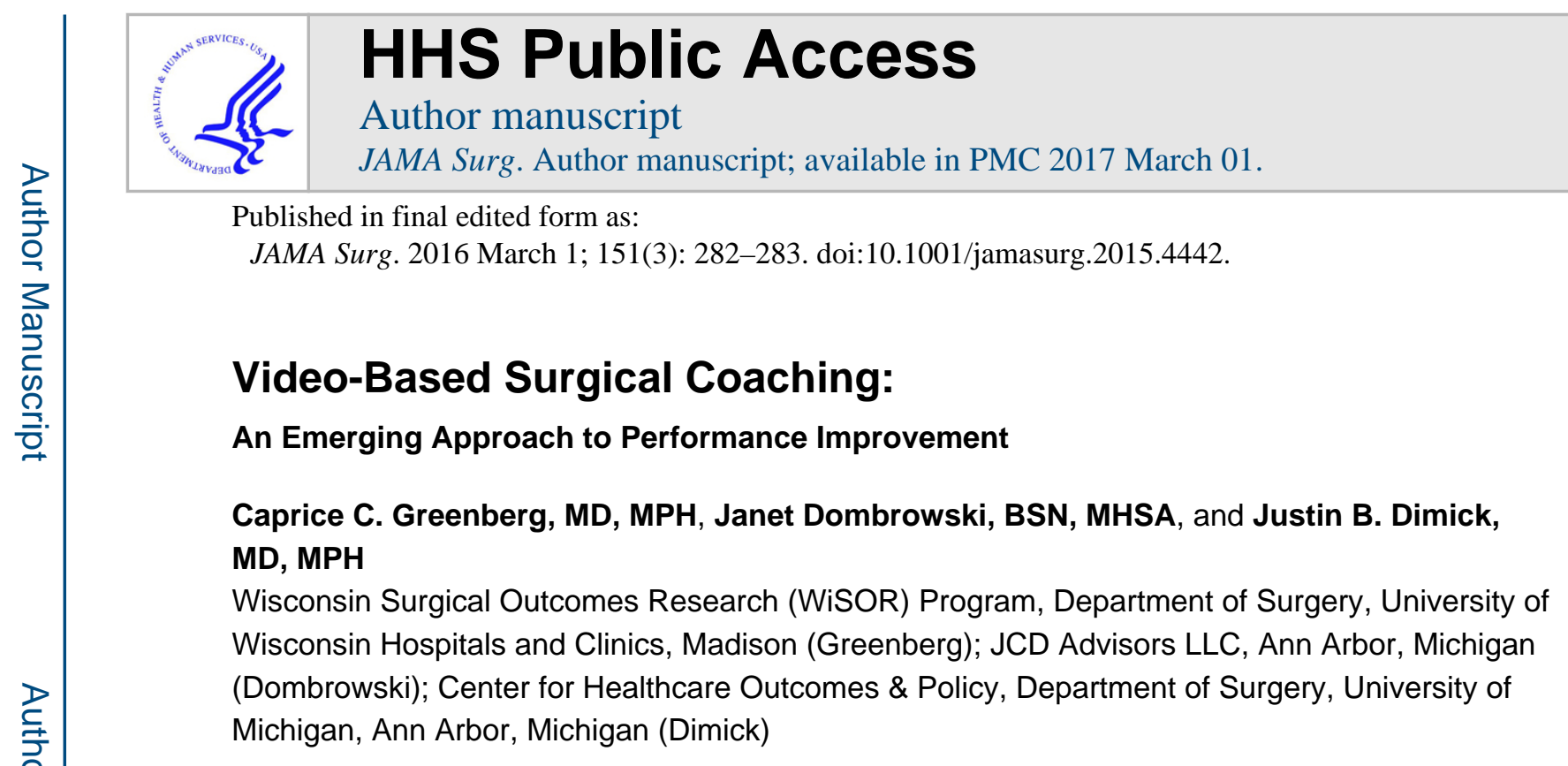

\title{
What Is the Innovation?
}

Surgeons are committed to optimizing the quality and safety of the care that we provide to ensure the best outcomes for our patients. Most current quality improvement initiatives target the system and processes of care around us. In contrast, surgical training emphasizes the primacy of the surgeon in determining the outcomes of our patients. As a result, many surgeons struggle with the current focus on a systems view of quality and safety and the apparent incongruity with the traditional surgical value of personal responsibility. Despite the focus placed on personal performance during residency, once training is complete, there is a lack of structured programs to support individual surgeons in examining their skills and their own individual improvement.

Several ongoing studies are evaluating an innovation in continuing professional development-a structured approach to peer coaching based on regular facilitated videobased review of operative performance. Video-based peer coaching matches an individual surgeon in community or academic practice with a surgical colleague who has been trained in the core principles of coaching. The pair uses a video-recorded operation by the participating surgeon to examine operative performance including technical, cognitive, and interpersonal skills. During a series of 1-hour coaching sessions, they identify individualized performance goals, evaluate current performance, and design an action plan to advance

\footnotetext{
Corresponding Author: Caprice C. Greenberg, MD, MPH, Wisconsin Surgical Outcomes Research (WiSOR) Program, University of Wisconsin Hospitals and Clinics, 600 Highland Ave, Clinical Science Center, K6/100, Madison, WI 53792-1690 (greenberg@surgery.wisc.edu).

Conflict of Interest Disclosures: Dr Greenberg serves as a consultant to Johnson \& Johnson's Human Performance Institute. Dr Dombrowski is an employee of JCD Advisors, a sole proprietorship consulting company specializing in organizational and leadership development and executive/ physician coaching in healthcare. Dr Dimick is the cofounder of ArborMetrix, Inc, a company that makes software for measuring hospital quality and efficiency. No other disclosures were reported.

Role of the Funder/Sponsor: The funding agencies had no role in design and conduct of the study; collection, management, analysis, and interpretation of the data; preparation, review, or approval of the manuscript; and decision to submit the manuscript for publication.

Additional Contributions: We acknowledge and thank the Wisconsin Surgical Society and the Michigan Bariatric Surgery Collaborative for their participation in ongoing research that has informed the development of material presented in this article. We also acknowledge the contributions of the Wisconsin Surgical Coaching Program, including Hala Gousseini, PhD, Douglas Wiegmann, PhD, Heather Beasley, PhD, and Sudha Pavuluri Quamme, MD, MS, who were funded on both of the grants from the funding agencies.
} 
toward those goals. Such individualized peer performance assessment does not currently exist in surgery.

\section{What Are the Key Advantages Over Existing Approaches?}

In addition to targeting the system rather than individual practitioners, most existing quality improvement work in surgery focuses on optimizing perioperative processes rather than the operation itself. A landmark New England Journal of Medicine study provided great insight into the major impact of variation in individual surgeon intraoperative performance; Birkmeyer and colleagues ${ }^{1}$ demonstrated a significant linear relationship between surgeon technical skill (as rated by peer assessment of videos) and surgical outcomes. It stands to reason then that improving individual surgeon operative performance is a critical target to improve patient outcomes.

Our current approach to the education of practicing surgeons is not consistent with the ways in which adults acquire new knowledge. Most surgical posttraining learning activities such as conferences or postgraduate courses are didactic and rarely include individualized goaldirected learning. Educational activities are removed from the point of care and rely on the ability of individuals to independently acquire and transfer new knowledge into their clinical practice.

In a video-based coaching program, a surgeon works with a trained colleague to identify individual areas for improvement and assess overall performance. This offers not only a new, more effective approach to goal-directed learning, but also a novel approach to improving quality, safety, and outcomes.

\section{Is There Evidence Supporting the Benefits of the Innovation?}

Several projects are under way to investigate the benefits of surgical coaching for practicing surgeons; however, like all research, these results will not be available for some time. At this point, the existing evidence from other fields and for less experienced surgical learners suggests coaching is effective.

Several recent studies have investigated various forms of surgical coaching in students and residents in simulated settings with striking results. ${ }^{2-5}$ Each of these studies is a randomized clinical trial of undergraduate students, medical students, or residents where subjects are randomized to video-based coaching or traditional simulator training. All show highly significant improvement in operative performance as measured by general and procedurespecific instruments over the course of the study period. It will be important to see if similar results are achieved with more experienced surgeons and in the operating room rather than simulated settings.

\section{What Are the Barriers to Implementing This Innovation More Broadly?}

Mutabdzic and colleagues ${ }^{6}$ recently published a rigorous evaluation of the perception of practicing surgeons and found that surgical culture is the biggest impediment to implementation of surgical coaching. Many surgeons fear that surgical coaching programs 
will impede their autonomy and ability to set their own agenda for learning. This perception can be attributed to the common belief that surgical coaching is not innovative but a rebranding or euphemism for "mentoring," "advising," or "instructing," approaches to education and performance improvement we have been using for decades. Mutabdzic and colleagues offer a nuanced discussion of why this is not the case. They also identified concerns about image and authority, which should be mitigated by the approach described here of video-based performance review in a confidential setting removed from the point of care.

Successful participation in a coaching program requires challenging the collectively held mental models of competency and autonomy developed in traditional surgical training. We think of a "master surgeon" as one who has arrived at the highest level of performance, but this "expert" state of mind can stifle innovation and curiosity. In his book, The Fifth Discipline: The Art and Practice of the Learning Organization, ${ }^{7}$ Peter Senge describes personal mastery as a state of continual learning. He maintains that mastery is a process and life long discipline, not something that one possesses. Such thinking requires a paradigm shift for surgeons, one that focuses on formative evaluations in the pursuit of continual learning and perfection rather than summative evaluations that aim for competency. One of the key components of training for coaches in this intervention is to help them challenge this mental model in themselves and their coachees. Video-based peer coaching encourages surgeons to be continuous learners, challenging assumptions and approaches, and seek new and better ways of improving performance, quality, and outcomes.

Besides these cultural barriers, there are practical barriers to implementation. The most obvious is the lack of time to participate in surgical coaching. However, the intervention that we are currently investigating requires 1 hour 4 times per year-much less than is currently invested in other continuing medical education activities. Additionally, coaching programs will require development and oversight. It is currently unclear how these would be financed or organized if proven effective. Ongoing studies partner with state organizations, such as professional societies and quality collaboratives, which seem to be a natural fit (http:// www.surgery.wisc.edu/wscp; http://www.michiganbsc.org). National surgical specialty societies may also be interested in sponsoring such activities for their membership. We have also seen interest in development at the individual hospital and group practice level. Many of the concepts embodied by surgical coaching meet the requirements set by the American Board of Surgery for Maintenance of Certification. Given the resources that are currently invested in such activities, surgical coaching might be similarly supported in the future.

\section{In What Time Frame Will This Innovation Likely Be Applied Routinely?}

Video-based surgical coaching, or at least the principles and theories that it represents, has the potential to improve the quality and safety of surgical care. As the evidence surrounding its effectiveness accumulates, video-based coaching could play a key role inquality improvement, continuing professional education, hospital credentialing, and even board certification. Regardless of whether surgical coaching is the ultimate term used for these ideas, it is hard to argue with the underlying principles of continual practice improvement through formal peer-supported ongoing performance evaluation and practice adjustment. 
The timing seems right for the underlying shift in surgical culture this represents and such approaches are already being used in real-world settings.

\section{Acknowledgments}

Funding/Support: Funding was provided by the Wisconsin Partnership Program at the University of Wisconsin School of Medicine and Public Health and the National Institute of Health (National Institute of Diabetes and Digestive and Kidney Diseases, grant R01 DK101423-01).

\section{References}

1. Birkmeyer JD, Finks JF, O'Reilly A, et al. Michigan Bariatric Surgery Collaborative. Surgical skill and complication rates after bariatric surgery. N Engl J Med. 2013; 369(15):1434-1442. [PubMed: 24106936]

2. Singh P, Aggarwal R, Tahir M, Pucher PH, Darzi A. A randomized controlled study to evaluate the role of video-based coaching in training laparoscopic skills. Ann Surg. 2015; 261(5):862-869. [PubMed: 25185469]

3. Bonrath EM, Dedy NJ, Gordon LE, Grantcharov TP. Comprehensive surgical coaching enhances surgical skill in the operating room: a randomized controlled trial. Ann Surg. 2015; 262(2):205-212. [PubMed: 25822691]

4. Karam MD, Thomas GW, Koehler DM, et al. Surgical coaching from head-mounted video in the training of fluoroscopically guided articular fracture surgery. J Bone Joint Surg Am. 2015; 97(12): 1031-1039. [PubMed: 26085538]

5. Cole SJ, Mackenzie H, Ha J, Hanna GB, Miskovic D. Randomized controlled trial on the effect of coaching in simulated laparoscopic training. Surg Endosc. 2014; 28(3):979-986. [PubMed: 24196554]

6. Mutabdzic D, Mylopoulos M, Murnaghan ML, et al. Coaching surgeons: is culture limiting our ability to improve? Ann Surg. 2015; 262(2):213-216. [PubMed: 25894418]

7. Senge, P. The Fifth Discipline: The Art \& Practice of the Learning Organization. New York, NY: Doubleday; 2006. 